\title{
Erratum to: A robust semi-blind watermarking for color images based on multiple decompositions
}

\author{
Prasanth Vaidya S. ${ }^{1}$ Chandra Mouli P. V. S. S. R. ${ }^{1}$
}

Published online: 13 March 2017

(C) Springer Science+Business Media New York 2017

Erratum to: Multimed Tools Appl
DOI 10.1007/s11042-017-4355-0

In the original publication, the authors' photographs were interchanged. The original publication was corrected.

Chandra Mouli P. V. S. S. R.

chandramouli@vit.ac.in

Prasanth Vaidya S.

svprasanth.vaidya2014@vit.ac.in

1 School of Computing Science and Engineering VIT University, Vellore 632014, Tamil Nadu, India 